\title{
Utility of immunohistochemistry with C3d in C3 glomerulopathy
}

\author{
Malou L. H. Snijders $\mathbb{D}^{1} \cdot$ Bojou J. van de Wall-Neecke ${ }^{1} \cdot$ Dennis A. Hesselink ${ }^{2} \cdot$ Jan U. Becker ${ }^{3}$. \\ Marian C. Clahsen-van Groningen ${ }^{1}$
}

Received: 14 January 2019 / Revised: 30 July 2019 / Accepted: 30 July 2019 / Published online: 2 September 2019

(c) United States \& Canadian Academy of Pathology 2019

\begin{abstract}
C3-dominance by immunofluorescence is a defining feature in the diagnosis of $\mathrm{C} 3$ glomerulopathy. Most pathologists stain for $\mathrm{C} 3 \mathrm{c}$, which has been reported as a trace/negative even in otherwise clear-cut cases of dense deposit disease. We investigated the usefulness of C3d immunohistochemistry in biopsies with $\mathrm{C} 3$ glomerulopathy as an ancillary diagnostic tool. All biopsies from patients diagnosed with C3 glomerulopathy in the period January 2005 to June 2017 in the Erasmus MC, Rotterdam were included ( $n=14 ; 10 \mathrm{C} 3$ glomerulonephritis, 4 dense deposit disease). The staining pattern of C3d and $\mathrm{C} 4 \mathrm{~d}$ by immunohistochemistry was analyzed. As controls, biopsies from patients with immune complex membranoproliferative glomerulonephritis $(n=2)$, infection-associated glomerulonephritis $(n=6)$, pauci-immune crescentic glomerulonephritis $(n=7)$, tubulointerstitial nephritis $(n=7)$ and chronic-active antibody-mediated rejection $(n=9)$ were included. All 14 biopsies with C3 glomerulopathy showed a C3d score of $\geq 2$, including two clear-cut biopsies with C3 glomerulopathy originally showing a trace/negative staining for C3c. In the control group, a C3d score $\geq 2$ was observed in 11 biopsies (35\%; 2 with immune complex membranoproliferative glomerulonephritis (100\%), 6 with infection-associated glomerulonephritis (100\%), 1 with pauci-immune crescentic glomerulonephritis (14\%), 1 with tubulointerstitial nephritis $(14 \%)$ and 1 with chronic-active antibody-mediated rejection (11\%)). C4d was positive in $71 \%$ of the biopsies with $\mathrm{C} 3$ glomerulopathy (10/14). In conclusion, C3d immunohistochemistry is a valuable tool in the diagnosis of C3 glomerulopathy, especially in cases in which $\mathrm{C} 3 \mathrm{c}$ immunofluorescence shows a trace/negative. We recommend the use of $\mathrm{C} 3 \mathrm{~d}$ in addition to $\mathrm{C} 3 \mathrm{c}$ in cases suspicious for $\mathrm{C} 3$ glomerulopathy.
\end{abstract}

\section{Introduction}

C3 glomerulopathy is a rare type of glomerulonephritis that encompasses both $\mathrm{C} 3$ glomerulonephritis and dense deposit disease [1]. C3 glomerulopathy is characterized by $\mathrm{C} 3$ deposits in the glomeruli and is caused by uncontrolled

These authors contributed equally: Jan U. Becker, Marian C. Clahsenvan Groningen

Malou L. H. Snijders

m.snijders@erasmusmc.nl

1 Department of Pathology, Erasmus MC, University Medical Center Rotterdam, Rotterdam, The Netherlands

2 Department of Internal Medicine, Division of Nephrology and Renal Transplantation, Erasmus MC, University Medical Center Rotterdam, Rotterdam, The Netherlands

3 Institute of Pathology, University Hospital of Cologne, Cologne, Germany activation of the alternative pathway of the complement system [2,3]. When the alternative complement pathway becomes activated, $\mathrm{C} 3$ is split into $\mathrm{C} 3 \mathrm{a}$ and $\mathrm{C} 3 \mathrm{~b}$ by $\mathrm{C} 3$ convertase. $\mathrm{C} 3 \mathrm{~b}$ can react with other components of the complement cascade leading to the formation of the membrane attack complex (C5-C9), which together with C3a induces localized cell injury and inflammation. Degradation of $\mathrm{C} 3 \mathrm{~b}$ leads to the formation of $\mathrm{C} 3 \mathrm{c}$ and the end product C3d [4-6].

Activation of the alternative complement pathway in patients with $\mathrm{C} 3$ glomerulopathy can be caused by mutations in the complement genes, regulatory factors or by acquired defects [7]. For example, specific autoantibodies called C3 nephritic factor, inhibitory autoantibodies against complement factor $\mathrm{H}$ as well as inhibitory genetic alterations in the complement factor $\mathrm{H}$ gene can impair normal regulation of the alternative complement system [8-11]. Rarely, monoclonal immunoglobulin may cause C3 glomerulopathy [12].

Renal biopsies from patients with C3 glomerulopathy can reveal various patterns of glomerular injury by light 
microscopy. In rare cases, no abnormalities are visualized by light microscopy [3]. The main feature of $\mathrm{C} 3$ glomerulopathy is the presence of isolated $\mathrm{C} 3$ deposits in the glomeruli [2, 3]. In 2013, the definition of C3 glomerulopathy was further defined by the presence of dominant C3 staining with a staining score being at least two orders of magnitude greater than the other stainings (i.e. IgG, IgM, IgA and C1q) [3, 13]. Based on electron microscopy findings, C3 glomerulopathy can be further subclassified into C3 glomerulonephritis and dense deposit disease, the prototypical form of $\mathrm{C} 3$ glomerulopathy [2].

In most laboratories, C3 is detected by immunofluorescence on frozen kidney tissue using an antibody against $\mathrm{C} 3 \mathrm{c}[3,14]$. However, even in clear-cut cases of dense deposit disease, $\mathrm{C} 4 \mathrm{~d}$ has been shown to be dominant on immunostaining instead of $\mathrm{C} 3 \mathrm{c}$ in the experience of others $[15,16]$, as well as in our own experience. $\mathrm{C} 4 \mathrm{~d}$ is a fragment of $\mathrm{C} 4$ that can be derived from activation of both the classical and lectin complement pathway [17]. In contrast, the activation of $\mathrm{C} 4$ is bypassed in the alternative pathway of complement [18]. After the activation of C4, $\mathrm{C} 4 \mathrm{~b}$ is generated which is then cleaved into $\mathrm{C} 4 \mathrm{~d}$ and other fragments such as $\mathrm{C} 4 \mathrm{c}[17,19]$.

C $3 \mathrm{~d}$ has been suggested to be a more sensitive marker to detect complement activation than the currently used immunofluorescence antibody to C3c [20]. While C3c and other components of the complement system disappear after recovery from cell injury, $\mathrm{C} 3 \mathrm{~d}$ remains attached to the target cell [20]. Therefore, C3d might be a more robust marker of C3 activation than C3c. Moreover, recent mass spectrometry data have shown that $\mathrm{C} 3 \mathrm{~b}$, which also covalently binds surrounding structures, and $\mathrm{C} 3 \mathrm{~d}$ rather than $\mathrm{C} 3 \mathrm{c}$, accumulate in $\mathrm{C} 3$ glomerulopathy [21].

These observations challenge the traditional use of an antibody against $\mathrm{C} 3 \mathrm{c}$ as the sole means of detecting $\mathrm{C} 3$ activation and deposition. In the present study we investigated if C3d staining by immunohistochemistry could be a useful complementary tool for detecting $\mathrm{C} 3$ deposits in the diagnosis of $\mathrm{C} 3$ glomerulopathy, particularly in those cases that are pathogenetically defined but do not meet the consensus criterion of C3-dominance with the traditionally used $\mathrm{C} 3 \mathrm{c}$ immunofluorescence.

\section{Methods}

\section{Study cohort}

The database of the Department of Pathology (Erasmus MC, University Medical Center, Rotterdam, the Netherlands) was searched retrospectively for biopsies from patients diagnosed with C3 glomerulopathy between January 2005 and June 2017. The following clinical data were collected: age, gender, serum creatinine, proteinuria, $\mathrm{C}$-reactive protein and serum $\mathrm{C} 3$ and $\mathrm{C} 4$ concentrations at the time of biopsy. The presence of antinuclear antigen antibodies, antineutrophil cytoplasmic antibodies, double stranded DNA antibodies, C3 nephritic factor autoantibodies and complement factor $\mathrm{H}$ autoantibodies, and genetic mutations in the complement genes was evaluated within 1 month before to 1 month after the first biopsy.

\section{Cases}

A total of 14 biopsies from 11 different patients with C3 glomerulopathy were identified; eight patients were diagnosed with $\mathrm{C} 3$ glomerulonephritis and three with dense deposit disease. In two patients, more than one biopsy was performed (Table 1). In patient 11 , two biopsies were performed: the first biopsy did not show specific glomerular deposition for $\mathrm{C} 3 \mathrm{c}$ by immunofluorescence. Therefore, a second biopsy was performed 6 weeks later. In patient 8 , four biopsies were performed; the first biopsy was a native kidney biopsy that showed C3 glomerulonephritis. Four years later the patient received a kidney transplant with a decline of renal function 2 weeks after transplantation. A total of three allograft biopsies was subsequently performed ( 2 weeks, 8 months and 13 months after transplantation, respectively) which all showed features of a recurrence of C3 glomerulonephritis. The renal allograft biopsy taken 13 months after transplantation also showed light microscopic features of a borderline acute cellular rejection and was therefore excluded from the present study.

\section{Controls}

Two biopsies from patients with immune complex membranoproliferative glomerulonephritis (both cases of hepatitis $\mathrm{C}$-associated immune complex membranoproliferative glomerulonephritis), six biopsies from patients with infectionassociated glomerulonephritis, seven biopsies from patients with pauci-immune crescentic glomerulonephritis, seven biopsies from patients with tubulointerstitial nephritis and nine from patients with chronic-active antibody-mediated rejection with transplant glomerulopathy (Banff Lesion Score $\mathrm{cg}>0$ ) were included as controls [22].

\section{Tissue processing}

For light microscopy, paraffin-embedded sections were routinely stained with hematoxylin and eosin, periodic acidSchiff, Jones and Trichrome, according to the standardized diagnostic protocols for renal biopsy processing. Immunofluorescence staining for $\operatorname{IgG}, \operatorname{IgA}, \operatorname{IgM}, \mathrm{C} 3 \mathrm{c}, \mathrm{C} 1 \mathrm{q}$, kappa and lambda on fresh snap-frozen renal tissue was routinely performed immediately after renal biopsy in all cases. 


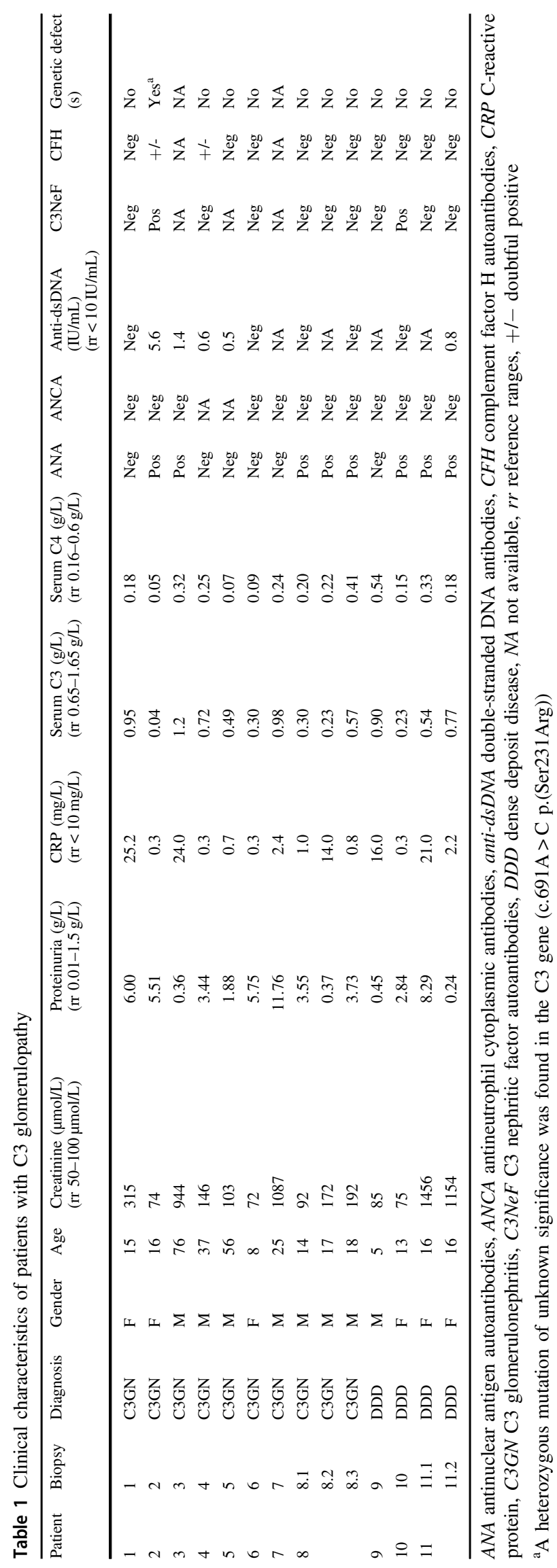

Immunofluorescence was performed on $5 \mu \mathrm{m}$ sections of fresh snap-frozen tissue, which were air dried on adhesive glass slides and pretreated with acetone for $10 \mathrm{~min}$. Fluorescein-tagged polyclonal rabbit antihuman antibodies to $\mathrm{IgG}, \mathrm{Ig} \mathrm{A}, \mathrm{IgM}, \mathrm{C} 3 \mathrm{c}, \mathrm{C} 1 \mathrm{q}$, kappa and lambda were all performed on a VENTANA BenchMark ULTRA according to the BenchMark Ultra protocol. For C3c, incubation with anti-C3c-FITC conjugated (Ventana 760-2686) for $24 \mathrm{~min}$ at $36^{\circ} \mathrm{C}$ was performed. Immunofluorescence results, including $\mathrm{C} 3 \mathrm{c}$, were collected retrospectively from the biopsy reports. Electron microscopy was performed in all cases to differentiate between $\mathrm{C} 3$ glomerulonephritis and dense deposit disease.

For the purpose of the present study, immunohistochemical C3d staining was performed on $2-3 \mu \mathrm{m}$ sections of formalin-fixed paraffin-embedded tissue. Tissue sections were dried overnight at $58{ }^{\circ} \mathrm{C}$ and subsequently deparaffinized, rehydrated and subjected to antigen retrieval using Lab Vision ${ }^{\mathrm{TM}}$ PT Module ${ }^{\mathrm{TM}}$ Deparaffinization and Heat-Induced Epitope Retrieval Solutions at $\mathrm{pH}$ 8. The tissue samples were incubated with the polyclonal antibody against C3d (\#403A-76, Cell Marque) at $97{ }^{\circ} \mathrm{C}$ for $20 \mathrm{~min}$ and dilution 1:50. The detection system was used as recommended by the manufacturer.

Immunohistochemical $\mathrm{C} 4 \mathrm{~d}$ staining was performed on $4 \mu \mathrm{m}$ slides of formalin-fixed paraffin-embedded tissue with an automated, validated and accredited staining system (Ventana Benchmark ULTRA, Ventana Medical Systems, Tucsen, AZ, USA) using an ultra-view universal DAB detection kit. In brief, following deparaffinization and heatinduced antigen retrieval with CC1 (\#950-124, Ventana) for $64 \mathrm{~min}$, the tissue samples were incubated with C4d (SP91, \#760-4803, Cell Marque) for $24 \mathrm{~min}$ at $36^{\circ} \mathrm{C}$. C3d and C4d immunohistochemistry were scored on a scale from 0 to $3+$ by two pathologists (MS and JUB) who were blinded to patient information.

We ruled out the entity of masked IgG kappa deposits recently described by Larsen et al. [23, 24] by staining all biopsies for kappa-light chains by immunohistochemistry after proteinase treatment of paraffin sections. Five biopsies from patients with $\mathrm{C} 3$ glomerulopathy showed positivity for kappa (36\%); three biopsies showed 2+ staining (21\%) and two biopsies $1+$ staining (14\%). 3+ staining for kappa was not observed in any of the biopsies with $\mathrm{C} 3$ glomerulopathy, effectively ruling out this entity in all biopsies.

\section{Results}

\section{Patient characteristics}

Patient characteristics are given in Table 1 . The mean age at the time of diagnosis was $26 \pm 22$ years (range 5-76 years). 
Six patients $(55 \%)$ were male. The mean serum creatinine concentration at the time of diagnosis was $412 \pm$ $151 \mu \mathrm{mol} / \mathrm{L}$. The degree of proteinuria at the time of biopsy was highly variable and ranged from 0.4 to $11.8 \mathrm{~g} / \mathrm{L}$ (mean proteinuria $4.5 \pm 3.4 \mathrm{~g} / \mathrm{L}$ ). Nephrotic-range proteinuria was present in 6 of 11 patients. C-reactive protein was relatively low in all patients (mean $10.1 \mathrm{mg} / \mathrm{L}$, range $0.30-25.2 \mathrm{mg} / \mathrm{L}$; reference range $<10 \mathrm{mg} / \mathrm{L}$ ). Mean serum $\mathrm{C} 3$ at the time of diagnosis was $0.51 \mathrm{~g} / \mathrm{L}$ (range $0.04-0.98 \mathrm{~g} / \mathrm{L}$; reference range: $0.65-1.65 \mathrm{~g} / \mathrm{L}$ ) and a low serum C3 $(<0.65 \mathrm{~g} / \mathrm{L})$ was observed in six patients. Mean serum $\mathrm{C} 4$ at the time of diagnosis was $0.22 \mathrm{~g} / \mathrm{L}$ (range $0.05-0.54 \mathrm{~g} / \mathrm{L}$; reference range: $0.16-0.6 \mathrm{~g} / \mathrm{L}$ ); a decreased serum $\mathrm{C} 4$ was observed in four patients. Antinuclear antigen antibodies were found in five patients (45\%); none of the patients tested positive for antineutrophil cytoplasmic antibodies. Evaluation of the alternative complement pathway was performed in nine patients. C3 nephritic factor autoantibodies were present in two patients (patients 2 and 10; Table 1). In one patient (patient 2) a heterozygous mutation with unknown significance was found in the $\mathrm{C} 3$ gene ((c.691A > C p.(Ser231Arg)). Complement factor $\mathrm{H}$ autoantibodies were dubiously positive in two patients (patients 2 and 4).

\section{Glomerular C3c staining by IF}

Dominant $\mathrm{C} 3 \mathrm{c}$ staining by immunofluorescence was observed in 12 biopsies from patients with $\mathrm{C} 3$ glomerulopathy (ten biopsies showed $3+$ staining and two biopsies $2+$ staining). In two biopsies (patients 2 and 11) C3c by immunofluorescence showed negative to $1+$ staining only (Fig. 1). C3c by immunofluorescence was also dominant with $3+$ in all biopsies with infection-associated glomerulonephritis.

\section{Immunohistochemical glomerular C3d staining}

All 14 biopsies with $\mathrm{C} 3$ glomerulopathy were positive for C3d: 11 biopsies showed 3+ staining for C3d and three biopsies showed $2+$ staining for C3d (Fig. 2). A score of $2+$ and $3+$ was observed in the two biopsies showing $1+$ and negative $\mathrm{C} 3 \mathrm{c}$ staining by immunofluorescence, respectively (Table 2).

In the control group, C3d immunohistochemistry was positive in 22 of 31 biopsies; $2+$ or $3+\mathrm{C} 3 \mathrm{~d}$ staining was observed in 11 of 31 biopsies (35\%): two biopsies with immune complex membranoproliferative glomerulonephritis (100\%), six biopsies with infection-associated
Fig. 1 Examples of $\mathrm{C} 3 \mathrm{c}$ by immunofluorescence in biopsies from patients with $\mathrm{C} 3$ glomerulopathy (negative (a; biopsy 2), $1+$ (b; biopsy 11.1), $2+(\mathbf{c}$; biopsy 5$)$ and $3+(\mathbf{d}$; biopsy 8.3)
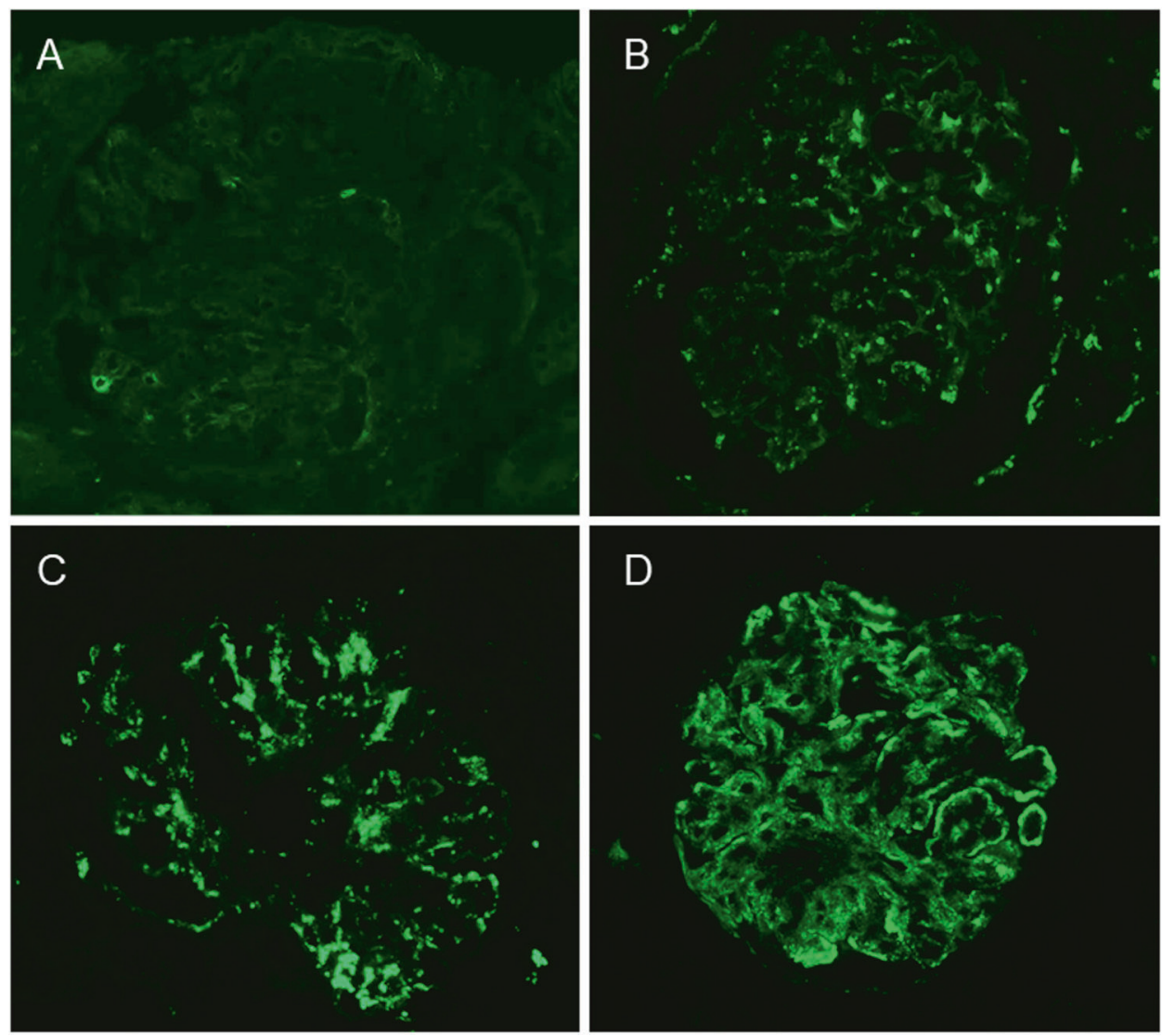

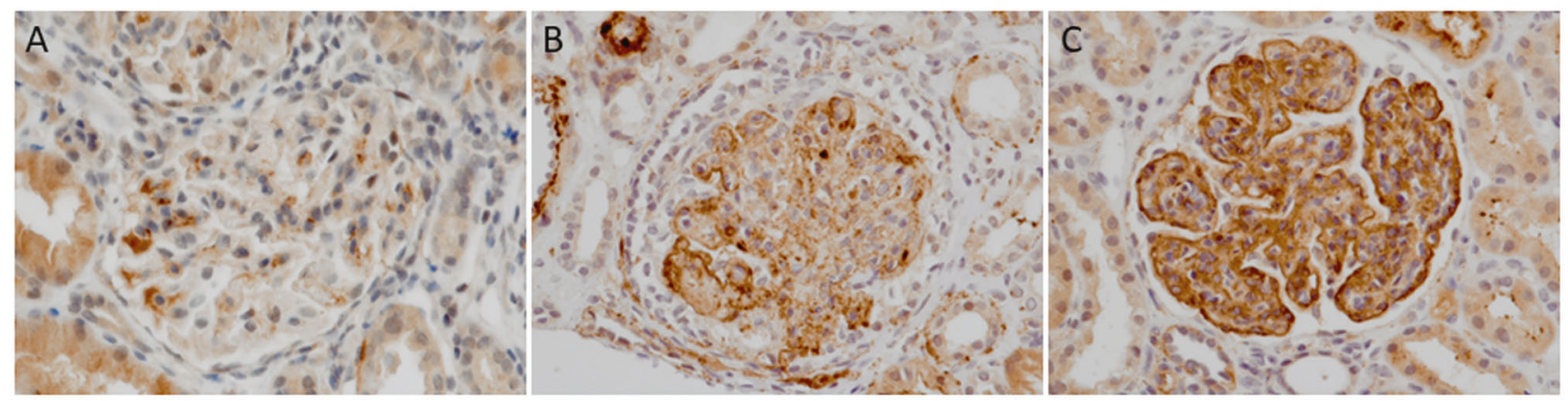

Fig. 2 Examples of C3d immunohistochemistry 1+ (a; biopsy 23), 2+ (b; biopsy 11.1) and 3+ (c; biopsy 2)

Table 2 Overview of C3c staining score by immunofluorescence and C3d staining by immunohistochemistry in biopsies from patients with C3 glomerulopathy

\begin{tabular}{lllll}
\hline Patient & Biopsy & Diagnosis & C3c IF & C3d IHC \\
\hline 1 & 1 & C3GN & 3 & 3 \\
2 & 2 & C3GN & 0 & 3 \\
3 & 3 & C3GN & 3 & 3 \\
4 & 4 & C3GN & 3 & 3 \\
5 & 5 & C3GN & 2 & 3 \\
6 & 6 & C3GN & 3 & 3 \\
7 & 7 & C3GN & 3 & 2 \\
8 & 8.1 & C3GN & 3 & 3 \\
& 8.2 & C3GN & NA & 3 \\
& 8.3 & C3GN & 3 & 3 \\
9 & 9 & DDD & 3 & 3 \\
10 & 10 & DDD & 3 & 3 \\
11 & 11.1 & DDD & 1 & 2 \\
& 11.2 & DDD & 2 & 2 \\
\hline
\end{tabular}

C3GN C3 glomerulonephritis, $D D D$ dense deposit disease, $N A$ not available

${ }^{\mathrm{a}}$ No glomeruli present in the snap frozen renal biopsy

glomerulonephritis (100\%), one biopsy with pauci-immune crescentic glomerulonephritis (14\%), one biopsy with tubulointerstitial nephritis (14\%) and one biopsy with chronic-active antibody-mediated rejection (11\%). A score of $1+$ for $\mathrm{C} 3 \mathrm{~d}$ was seen in 11 of 31 biopsies (35\%): four biopsies with pauci-immune crescentic glomerulonephritis (57\%), one biopsy with tubulointerstitial nephritis (14\%), six biopsies with chronic-active antibody-mediated rejection $(67 \%)$. The immunohistochemical findings are shown in Table 3.

\section{Consensus criteria for C3 glomerulopathy}

Ten of 14 biopsies fulfilled the consensus criteria for C3 glomerulopathy based on $\mathrm{C} 3 \mathrm{c}$ immunofluorescence staining alone (8/10 C3 glomerulonephritis and 2/4 dense deposit disease) [3]. These ten biopsies showed dominant $\mathrm{C} 3 \mathrm{c}$ staining by immunofluorescence and electron-dense deposits without substructure by electron microscopy.

Based on both $\mathrm{C} 3 \mathrm{c}$ immunofluorescence and $\mathrm{C} 3 \mathrm{~d}$ immunohistochemistry, 12 of 14 biopsies (10/10 C3 glomerulonephritis, 2/4 dense deposit disease) fulfilled the criteria for $\mathrm{C} 3$ glomerulopathy. In patient 2, the diagnosis of $\mathrm{C} 3$ glomerulopathy could only be made after $\mathrm{C} 3 \mathrm{~d}$ immunohistochemistry staining because $\mathrm{C} 3 \mathrm{c}$ immunofluorescence was negative while $\mathrm{C} 3 \mathrm{~d}$ immunohistochemistry showed $3+$ staining. This patient showed characteristic ribbon-like electron-dense deposits in the thickened glomerular basement membrane by electron microscopy. One of the three biopsies performed in patient 8 did not contain glomeruli in the snap-frozen tissue and therefore the diagnosis of $\mathrm{C} 3$ glomerulopathy could not be made on this biopsy based on $\mathrm{C} 3 \mathrm{c}$ immunofluorescence. However, C3d immunohistochemistry on the formalin-fixed paraffin-embedded slide showed $3+$ staining in this biopsy. In patient 11 , the diagnosis of $\mathrm{C} 3$ glomerulopathy could not be made according to the consensus criteria using both $\mathrm{C} 3 \mathrm{c}$ immunofluorescence and C3d immunohistochemistry. However, electron microscopy showed glomerular basement membrane thickening with dense osmiophilic intramembranous electron deposits diagnostic of dense deposit disease. In this patient, two biopsies were performed, showing $1+$ and $2+$ staining for $\mathrm{C} 3 \mathrm{c}$ immunofluorescence respectively, whereas both biopsies showed $2+$ staining for $\mathrm{C} 3 \mathrm{~d}$ immunohistochemistry, raising the $\mathrm{C} 3$ staining to codominant. $\mathrm{C} 1 \mathrm{q}$ showed $2+$ staining in both biopsies.

\section{Immunohistochemical glomerular C4d staining}

C4d staining was positive in 10 of 14 biopsies with C3 glomerulopathy (71\%); six biopsies showed $3+$ staining (43\%), three biopsies $2+$ staining $(21 \%)$ and one biopsy $1+$ staining (7\%) (Fig. 3). In addition, both biopsies with immune complex membranoproliferative glomerulonephritis showed $3+$ staining for $\mathrm{C} 4 \mathrm{~d}(100 \%)$. Only $2 / 6$ biopsies with infection-associated glomerulonephritis showed $\geq 2+$ staining for $\mathrm{C} 4 \mathrm{~d}(33 \%)$, the other four were negative. Three biopsies 
Table 3 Immunohistochemical staining with C3d, C4d and kappa in biopsies from patients with C3 glomerulonephritis, dense deposit disease, immune complex membranoproliferative glomerulonephritis, infection-associated glomerulonephritis, pauciimmune glomerulonephritis, tubulointerstitial nephritis and chronicactive antibody-mediated rejection

\begin{tabular}{|c|c|c|c|c|c|}
\hline Patient & Biopsy & Diagnosis & C3d & $\mathrm{C} 4 \mathrm{~d}$ & kappa \\
\hline 1 & 1 & C3GN & 3 & 3 & 0 \\
\hline 2 & 2 & C3GN & 3 & 2 & 0 \\
\hline 3 & 3 & C3GN & 3 & 0 & 2 \\
\hline 4 & 4 & $\mathrm{C} 3 \mathrm{GN}$ & 3 & 1 & 0 \\
\hline 5 & 5 & C3GN & 3 & 3 & 0 \\
\hline 6 & 6 & C3GN & 3 & 2 & 0 \\
\hline 7 & 7 & $\mathrm{C} 3 \mathrm{GN}$ & 2 & 3 & 0 \\
\hline \multirow[t]{3}{*}{8} & 8.1 & $\mathrm{C} 3 \mathrm{GN}$ & 3 & 3 & 0 \\
\hline & 8.2 & $\mathrm{C} 3 \mathrm{GN}$ & 3 & 2 & 2 \\
\hline & 8.3 & C3GN & 3 & 0 & 1 \\
\hline 9 & 9 & DDD & 3 & 0 & 0 \\
\hline 10 & 10 & DDD & 3 & 3 & 2 \\
\hline \multirow[t]{2}{*}{11} & 11.1 & DDD & 2 & 0 & 0 \\
\hline & 11.2 & DDD & 2 & 3 & 1 \\
\hline 12 & 12 & ICMGNP & 3 & 3 & 1 \\
\hline 13 & 13 & ICMGNP & 3 & 3 & 2 \\
\hline 14 & 14 & IAGN & 3 & 3 & 0 \\
\hline 15 & 15 & IAGN & 3 & 2 & 0 \\
\hline 16 & 16 & IAGN & 3 & 0 & 0 \\
\hline 17 & 17 & IAGN & 3 & 0 & 0 \\
\hline 18 & 18 & IAGN & 3 & 0 & 0 \\
\hline 19 & 19 & IAGN & 3 & 0 & 0 \\
\hline 20 & 20 & PCGN & 1 & 0 & 1 \\
\hline 21 & 21 & PCGN & 0 & 0 & 1 \\
\hline 22 & 22 & PCGN & 1 & 2 & 0 \\
\hline 23 & 23 & PCGN & 1 & 0 & 0 \\
\hline 24 & 24 & PCGN & 2 & 2 & 3 \\
\hline 25 & 25 & PCGN & 1 & 2 & 0 \\
\hline 26 & 26 & PCGN & 0 & 0 & 0 \\
\hline 27 & 27 & TIN & 0 & 0 & 0 \\
\hline 28 & 28 & TIN & 0 & 0 & 0 \\
\hline 29 & 29 & TIN & 0 & 0 & 0 \\
\hline 30 & 30 & TIN & 0 & 0 & 0 \\
\hline 31 & 31 & TIN & 2 & 0 & 0 \\
\hline 32 & 32 & TIN & 0 & 0 & 0 \\
\hline 33 & 33 & TIN & 1 & 0 & 0 \\
\hline 34 & 34 & c-aABMR & 0 & 3 & 0 \\
\hline 35 & 35 & c-aABMR & 1 & 1 & 0 \\
\hline 36 & 36 & c-aABMR & 1 & 0 & 0 \\
\hline 37 & 37 & $\mathrm{c}-\mathrm{aABMR}$ & 0 & 0 & 1 \\
\hline 38 & 38 & $\mathrm{c}-\mathrm{aABMR}$ & 1 & 1 & 0 \\
\hline 39 & 39 & c-aABMR & 1 & 1 & 1 \\
\hline 40 & 40 & $\mathrm{c}-\mathrm{aABMR}$ & 1 & 2 & 0 \\
\hline 41 & 41 & c-aABMR & 2 & 1 & 0 \\
\hline 42 & 42 & c-aABMR & 1 & 0 & 0 \\
\hline
\end{tabular}

$c$ - $a A B M R$ chronic-active antibody-mediated rejection, $C 3 G N \mathrm{C} 3$ glomerulonephritis, $D D D$ dense deposit disease, IAGN infection-associated glomerulonephritis, ICMGNP immune complex membranoproliferative glomerulonephritis, $P C G N$ pauci-immune glomerulonephritis, TIN tubulointerstitial nephritis with pauci-immune crescentic glomerulonephritis showed $2+$ staining $(43 \%)$, while the other four were negative for C4d (57\%). All seven biopsies with tubulointerstitial nephritis were negative for $\mathrm{C} 4 \mathrm{~d}$. In biopsies with chronicactive antibody-mediated rejection, one showed $3+$ staining $(11 \%)$, one showed $2+$ staining $(11 \%)$, four showed $1+$ staining (45\%) and three biopsies were negative (33\%).

\section{Discussion}

C3 glomerulopathy is a disease entity defined by dysregulation of the alternative complement pathway [2, 3]. This results in the deposition of complement $\mathrm{C} 3$ fragments in the glomerulus which is currently detected using immunofluorescence with an antibody to C3c [3]. However, cases of dense deposit disease, the prototypical form of C3 glomerulopathy, with $\mathrm{C} 4 \mathrm{~d}$-dominance (C4-dense deposit disease) not fulfilling the consensus criteria, have been observed [16, 25].

$\mathrm{C} 3 \mathrm{~d}$ is one of the final degradation products of $\mathrm{C} 3$ and is more stable in vivo than $\mathrm{C} 3 \mathrm{c}$, because $\mathrm{C} 3 \mathrm{~d}$ remains attached to the tissue site after recovery of injury leaving a visible footprint [20]. Therefore, we hypothesized that C3d would be a more sensitive and robust immunostaining marker for the diagnosis of $\mathrm{C} 3$ glomerulopathy than $\mathrm{C} 3 \mathrm{c}$.

We observed at least $2+$ staining for C3d in all 14 biopsies with $\mathrm{C} 3$ glomerulopathy. Interestingly, two biopsies with C3 glomerulopathy, in which C3c staining by immunofluorescence was negative/sparse, showed $\geq 2+$ staining with $\mathrm{C} 3 \mathrm{~d}$ by immunohistochemistry (patients 2 and 11), enabling the reclassification as at least $\mathrm{C} 3$-codominant.

In patient 11 , two biopsies were performed in which the diagnosis of $\mathrm{C} 3$ glomerulopathy, according to the consensus criteria, could still not be made after C3d immunohistochemistry since both $\mathrm{C} 3 \mathrm{~d}$ immunohistochemistry and $\mathrm{C} 1 \mathrm{q}$ immunofluorescence showed $2+$ staining in both biopsies [3]. However, electron microscopy showed glomerular basement membrane thickening with dense osmiophilic intramembranous deposits characteristic for dense deposit disease. So even after the use of C3d immunohistochemistry we are left with cases that, as either codominant or as only dominant by one magnitude do not fulfill the consensus criteria for C3 glomerulopathy. These findings raise the question whether the current consensus criteria are sensitive enough.

In patient $2, \mathrm{C} 4 \mathrm{~d}$ immunohistochemistry was also performed at the time of diagnosis. Sethi et al. recently described three cases with C3 glomerulopathy in which no or only sparse $\mathrm{C} 3 \mathrm{c}$ staining was observed by immunofluorescence and introduced a new entity called C4 glomerulopathy characterized by mesangial electron-dense deposits and bright staining for $\mathrm{C} 4 \mathrm{~d}$ with either 

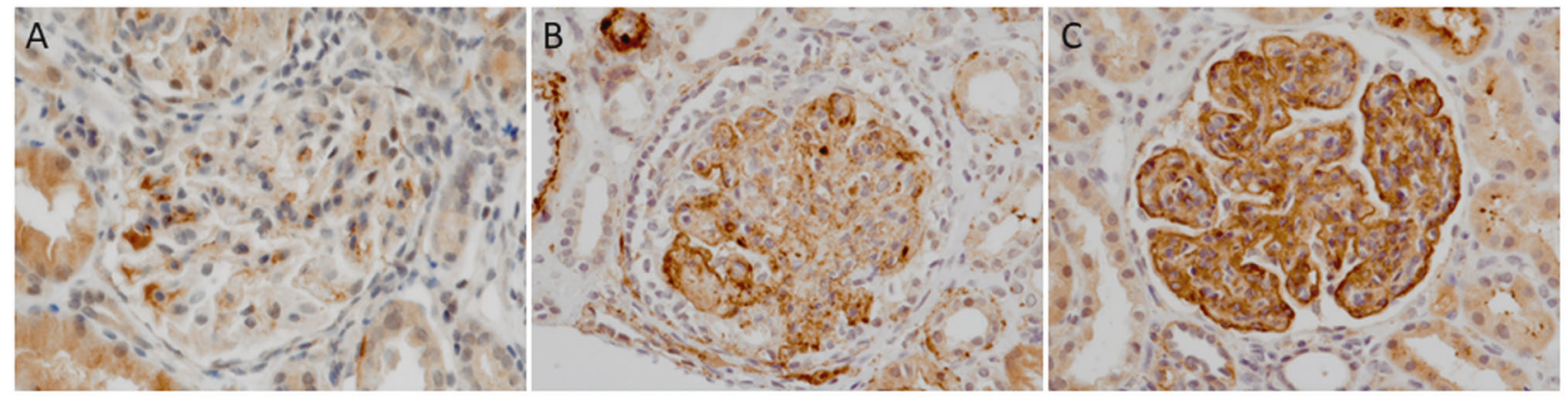

Fig. 3 Examples of C4d immunohistochemistry 1+ (a; biopsy 4), 2+ (b; biopsy 2) and 3+ (c; biopsy 11.2)

immunohistochemistry or immunofluorescence and absent to a trace staining for $\mathrm{C} 3$ and immunoglobulins $[15,16]$. In patient 2, C4d immunohistochemistry showed $2+$ staining and therefore the diagnosis of $\mathrm{C} 4$ glomerulopathy was made at that time. However, C3d staining by immunohistochemistry showed $3+$ staining and therefore this case might in fact represent a case of $\mathrm{C} 3$ glomerulopathy. Of course, we cannot rule out with absolute certainty a role for abnormal $\mathrm{C} 1$ or lectin pathway activation in this patient. However, positive $\mathrm{C} 3$ nephritic factor autoantibodies and a genetic mutation in the $\mathrm{C} 3$ gene were found, arguing for the diagnosis of $\mathrm{C} 3$ glomerulopathy in this particular case. Just relying on the traditional $\mathrm{C} 3 \mathrm{c}$ immunofluorescence along with $\mathrm{C} 4 \mathrm{~d}$ staining, cases might be misdiagnosed as $\mathrm{C} 4$ glomerulonephritis or $\mathrm{C} 4$ dense deposit disease. C3d immunohistochemistry could be helpful in avoiding this mistake and to demonstrate a histopathological correlate to C3 activation.

In a study by Sethi et al., the use of $\mathrm{C} 4 \mathrm{~d}$ was evaluated to distinguish C3 glomerulopathy from immune complex membranoproliferative glomerulonephritis [26]. They observed $\geq 2+$ C4d staining in $89 \%(16 / 18)$ of the biopsies with immune complex membranoproliferative glomerulonephritis, while C4d staining was found in only $20 \%$ (6/30) of the biopsies with C3 glomerulopathy showing only a trace or $1+$ staining. Based on these results, they suggested that negative glomerular staining for $\mathrm{C} 4 \mathrm{~d}$ can serve as a marker for C3 glomerulopathy. In contrast, we observed C4d immunohistochemical staining in 10/14 biopsies with $\mathrm{C} 3$ glomerulopathy (71\%), suggesting that $\mathrm{C} 4 \mathrm{~d}$ staining is in fact often observed in patients with $\mathrm{C} 3$ glomerulopathy. In line with our findings, Bouatou et al. concluded that $\mathrm{C} 4 \mathrm{~d}$ staining is of limited value for the discrimination between $\mathrm{C} 3$ glomerulopathy and immune complex membranoproliferative glomerulonephritis [27].

We observed the presence of both $\geq 2+$ staining for C3 (either $\mathrm{C} 3 \mathrm{c}$ or $\mathrm{C} 3 \mathrm{~d}$ ) and $\mathrm{C} 4 \mathrm{~d}$ in a total of nine biopsies with C3 glomerulopathy (64\%) showing evidence of a complement-mediated glomerular disease driven by both the alternative and classical/lectin pathway. This finding has been described previously in a single case of dense deposit disease by Vankalakunt et al. [28]. Singh et al. performed an observational study including 27 dense deposit disease cases and $14 \mathrm{C} 3$ glomerulonephritis cases. They observed C4d staining of variable intensity with $\geq 2+$ staining in $48 \%$ of dense deposit disease cases and $21 \%$ of $\mathrm{C} 3$ glomerulonephritis cases [29]. It has been hypothesized that in these cases the alternative complement pathway is upregulated in addition to activation of the classical or lectin pathway caused by infection, auto-immune disease or monoclonal gammopathy [18]. We stained for kappa after protease digestion on all biopsies and excluded monoclonal gammopathy in all C3 glomerulopathy cases in our study, effectively ruling out monoclonal gammopathy-associated forms of glomerulonephritis and the peculiar entity of masked kappa deposits recently described [23, 24]. The possibility of an infection as a trigger for $\mathrm{C} 3$ glomerulopathy in some of the patients reported here could not be totally excluded [30]. However, the rather low C-reactive protein concentration at the time of biopsy and the clinical findings in all patients argues against this.

The "typical" work-up for patients with suspicion for C3 glomerulopathy includes testing for antinuclear antigen antibodies, antineutrophil cytoplasmic antibodies, doublestranded DNA antibodies and exclusion of infections with hepatitis B and C, and HIV, as well as monoclonal gammopathy. In addition, patients are tested for serum complement $\mathrm{C} 3, \mathrm{C} 4$ and $\mathrm{C} 1 \mathrm{q}$, autoantibodies against complement factor $\mathrm{H}, \mathrm{C} 3$ nephritic factor, C4 nephritic factor, terminal complement complex, $\mathrm{Bb}, \mathrm{C} 3 \mathrm{bc}, \mathrm{C} 3 \mathrm{bBbP}$, Factor H, Factor I, Factor B and abnormalities in complement protein encoding or regulating genes.

Abnormalities in the alternative complement pathway are observed in most patients with C3 glomerulopathy [31, 32]. In contrast to other studies, no abnormalities in the alternative complement pathway were observed in the majority of C3 glomerulopathy patients in our study. This may be explained by the small cohort size or the fact that alternative complement pathway abnormalities were only thoroughly evaluated in 9 out 11 patients (82\%). 
Limitations of this proof-of-principle study are the small cohort size and the fact that this was a retrospective study and therefore some clinical data were not available. However, a relatively large control group was included to compare immunohistochemical $\mathrm{C} 3 \mathrm{~d}$ staining results in renal biopsies from patients with $\mathrm{C} 3$ glomerulopathy to biopsies from patients with such diverse diagnoses as immune complex membranoproliferative glomerulonephritis, infection-associated glomerulonephritis, pauci-immune crescentic glomerulonephritis, tubulointerstitial nephritis and chronic-active antibody-mediated rejection. As expected, biopsies from patients with immune complex membranoproliferative glomerulonephritis and infectionassociated glomerulonephritis showed $3+$ staining for $\mathrm{C} 3 \mathrm{~d}$ immunohistochemistry. Biopsies with tubulointerstitial nephritis, pauci-immune crescentic glomerulonephritis and chronic-active antibody-mediated rejection were negative or showed only $1+$ staining for C3d immunohistochemistry in most of the cases. C3 glomerulopathy can show overlapping histomorphologic features with pauci-immune crescentic glomerulonephritis and chronic-active antibody-mediated rejection which can make it difficult to distinguish between these entities by light microscopy [33-35]. Dominant C3d staining by immunohistochemistry may support the diagnosis of $\mathrm{C} 3$ glomerulopathy in these cases.

Based on the findings in the present study, we recommend the use of $\mathrm{C} 3 \mathrm{~d}$ immunohistochemistry in addition to $\mathrm{C} 3 \mathrm{c}$ immunofluorescence in all cases with membranoproliferative glomerulonephritis. In addition, both clinical suspicion and electron microscopy findings indicative of dense deposit disease could be of guidance to recommend the use of C3d immunohistochemistry, especially in those cases in which $\mathrm{C} 3 \mathrm{c}$ immunofluorescence shows a trace/ negative. Furthermore, C3d immunohistochemistry can be of value in those cases with a trace/negative $\mathrm{C} 3 \mathrm{c}$ immunofluorescence and positive $\mathrm{C} 4 \mathrm{~d}$ staining, since these cases could be incorrectly labeled as C4d-dominant glomerulopathy. Also, in those cases where frozen renal tissue is not available, C3d immunohistochemistry could be used in common practice (in combination with $\mathrm{C} 3 \mathrm{c}$ immunohistochemistry when this is available). For example, in our study no glomeruli were present in a snap-frozen renal biopsy from patient 8 (biopsy 8.2). This is a problem in cases with a strong suspicion for $\mathrm{C} 3$ glomerulopathy. $\mathrm{C} 3 \mathrm{c}$ immunofluorescence has been reported to not be reliable by salvage technique on formalin-fixed paraffin-embedded tissue by some laboratories [36]. In those situations, C3d immunohistochemistry can be performed on the formalinfixed paraffin-embedded tissue.

In conclusion, C3d immunohistochemistry shows at least codominant staining in all biopsies with $\mathrm{C} 3$ glomerulopathy and could especially be helpful in those C3 glomerulopathy cases with absent or a trace $\mathrm{C} 3 \mathrm{c}$ staining by immunofluorescence. Therefore, we recommend the use of $\mathrm{C} 3 \mathrm{~d}$ in addition to $\mathrm{C} 3 \mathrm{c}$ on all biopsies suspicious for C3 glomerulopathy. Our results about the usefulness of C3d need validation in large, multicenter studies employing cluster-analysis approaches as has been suggested recently [37].

\section{Compliance with ethical standards}

Conflict of interest DAH has received lecture and consulting fees, as well as grant support from Astellas Pharma and Chiesi Farmacetici SpA, and grant support from Bristol Myers-Squibb. JUB received speaker honorarium and a research grant from Alexion Pharmaceuticals. The other authors declare no conflicts of interest.

Ethical approval The study protocol was consistent with international ethical and professional guidelines (the Declaration of Helsinki and the International Conference on Harmonization Guidelines for Good Clinical Practice). The study was approved by the local medical ethics committee (MEC-2016-350).

Publisher's note: Springer Nature remains neutral with regard to jurisdictional claims in published maps and institutional affiliations.

\section{References}

1. Barbour TD, Pickering MC, Terence Cook H. Dense deposit disease and C3 glomerulopathy. Semin Nephrol. 2013;33:493-507.

2. Fakhouri F, Frémeaux-Bacchi V, Noël LH, Cook HT, Pickering MC. C3 glomerulopathy: a new classification. Nat Rev Nephrol. 2010;6:494-9.

3. Pickering MC, D'Agati VD, Nester CM, Smith RJ, Haas M, Appel GB, et al. C3 glomerulopathy: consensus report. Kidney Int. 2013;84:1079-89.

4. Noris M, Remuzzi G. Glomerular diseases dependent on complement activation, including atypical hemolytic uremic syndrome, membranoproliferative glomerulonephritis, and C3 glomerulopathy: core curriculum 2015. Am J Kidney Dis. 2015;66:359-75.

5. Noris M, Remuzzi G. Overview of complement activation and regulation. Semin Nephrol. 2013;33:479-92.

6. Thurman JM, Holers VM. The central role of the alternative complement pathway in human disease. J Immunol. 2006;176:1305-10.

7. Zipfel PF, Skerka C, Chen Q, Wiech T, Goodship T, Johnson S, et al. The role of complement in $\mathrm{C} 3$ glomerulopathy. Mol Immunol. 2015;67:21-30.

8. Sethi S, Nester CM, Smith RJH. Membranoproliferative glomerulonephritis and C3 glomerulopathy: resolving the confusion. Kidney Int. 2012;81:434-41.

9. Salvadori M, Bertoni E. Complement related kidney diseases: recurrence after transplantation. World J Transpl. 2016;6: $632-45$.

10. de Córdoba SR, de Jorge EG. Translational mini-review series on complement factor $\mathrm{H}$ : genetics and disease associations of human complement factor H. Clin Exp Immunol. 2008;151:1-13.

11. Józsi M, Reuter S, Nozal P, López-Trascasa M, Sánchez-Corral P, Prohászka Z, et al. Autoantibodies to complement components in C3 glomerulopathy and atypical hemolytic uremic syndrome. Immunol Lett. 2014;160:163-71.

12. Sethi S, Fervenza FC, Rajkumar SV. Spectrum of manifestations of monoclonal gammopathy-associated renal lesions. Curr Opin Nephrol Hypertens. 2016;25:127-37. 
13. Hou J, Markowitz GS, Bomback AS, Appel GB, Herlitz LC, Barry Stokes M, et al. Toward a working definition of C3 glomerulopathy by immunofluorescence. Kidney Int. 2014;85:450-6.

14. West CD, Witte DP, McAdams AJ. Composition of nephritic factor-generated glomerular deposits in membranoproliferative glomerulonephritis type 2. Am J Kidney Dis. 2001;37:1120-30.

15. Sethi S, Quint PS, O'Seaghdha CM, Fervenza FC, Bijol V, Dorman A, et al. C4 Glomerulopathy: a disease entity associated with C4d deposition. Am J Kidney Dis. 2016;67:949-53.

16. Sethi S, Sullivan A, Smith RJ. C4 dense-deposit disease. N Engl J Med. 2014;370:784-6.

17. Murata K, Baldwin WM 3rd. Mechanisms of complement activation, C4d deposition, and their contribution to the pathogenesis of antibody mediated rejection. Transpl Rev (Orlando). 2009;23:139-50.

18. Angioi A, Fervenza FC, Sethi S, Zhang Y, Smith RJ, Murray D, et al. Diagnosis of complement alternative pathway disorders. Kidney Int. 2016;89:278-88.

19. Mortensen S, Kidmose RT, Petersen SV, Szilágyi Á, Prohászka Z, Andersen GR. Structural basis for the function of complement component $\mathrm{C} 4$ within the classical and lectin pathways of complement. J Immunol. 2015;194:5488-96.

20. Schulze M, Pruchno CJ, Burns M, Baker PJ, Johnson RJ, Couser WG. Glomerular $\mathrm{C} 3 \mathrm{c}$ localization indicates ongoing immune deposit formation and complement activation in experimental glomerulonephritis. Am J Pathol. 1993;142:179-87.

21. Sethi S, Vrana JA, Fervenza FC, Theis JD, Sethi A, Kurtin PJ, et al. Characterization of C3 in C3 glomerulopathy. Nephrol Dial Transpl. 2017;32:459-65.

22. Haas M, Loupy A, Lefaucheur C, Roufosse C, Glotz D, Seron D, et al. The Banff 2017 Kidney Meeting Report: revised diagnostic criteria for chronic active T cell-mediated rejection, antibody-mediated rejection, and prospects for integrative endpoints for next-generation clinical trials. Am J Transpl. 2018;18:293-307.

23. Larsen CP, Boils CL, Cossey LN, Sharma SG, Walker PD. Clinicopathologic features of membranous-like glomerulopathy with masked IgG kappa deposits. Kidney Int Rep. 2016;1: 299-305.

24. Larsen CP, Ambruzs JM, Bonsib SM, Boils CL, Cossey LN, Messias NC, et al. Membranous-like glomerulopathy with masked IgG kappa deposits. Kidney Int. 2014;86:154-61.

25. Ali A, Schlanger L, Nasr SH, Sethi S, Gorbatkin SM. Proliferative $\mathrm{C} 4$ dense deposit disease, acute thrombotic microangiopathy, a monoclonal gammopathy, and acute kidney failure. Am J Kidney Dis. 2016;67:479-82.

26. Sethi S, Nasr SH, De Vriese AS, Fervenza FC. C4d as a diagnostic tool in proliferative GN. J Am Soc Nephrol. 2015;26:2852-9.

27. Bouatou Y, Kers J, Chevalier-Florquin MSN, Claessen N, Nguyen TQ, Damman J, et al. Diagnostic accuracy of immunofluorescence versus immunoperoxidase staining to distinguish immune complexmediated glomerulonephritis and C3 dominant glomerulopathy. Histopathology. 2018;72:601-8.

28. Vankalakunti M, Augustine R, Jangamani R, Siddini V, Bonu R, Babu K, et al. Dense deposit disease involving C3 and C4d deposits. Indian J Nephrol. 2018;28:61-4.

29. Singh G, Singh SK, Nalwa A, Singh L, Pradeep I, Barwad A, et al. Glomerular C4d staining does not exclude a C3 glomerulopathy. Kidney Int Rep. 2019;4:698-709.

30. Sethi S, Fervenza FC, Zhang Y, Zand L, Meyer NC, Borsa N, et al. Atypical postinfectious glomerulonephritis is associated with abnormalities in the alternative pathway of complement. Kidney Int. 2013;83:293-9.

31. Iatropoulos P, Noris M, Mele C, Piras R, Valoti E, Bresin E, et al. Complement gene variants determine the risk of immunoglobulinassociated MPGN and C3 glomerulopathy and predict long-term renal outcome. Mol Immunol. 2016;71:131-42.

32. Noris M, Donadelli R, Remuzzi G. Autoimmune abnormalities of the alternative complement pathway in membranoproliferative glomerulonephritis and C3 glomerulopathy. Pedia Nephrol. 2019; 34:1311-23.

33. Singh L, Singh G, Bhardwaj S, Sinha A, Bagga A, Dinda A. Dense deposit disease mimicking a renal small vessel vasculitis. J Am Soc Nephrol. 2016;27:59-62.

34. Ito N, Ohashi R, Nagata M. C3 glomerulopathy and current dilemmas. Clin Exp Nephrol. 2017;21:541-51.

35. Remport A, Ivanyi B, Mathe Z, Tinckam K, Mucsi I, Molnar MZ. Better understanding of transplant glomerulopathy secondary to chronic antibody-mediated rejection. Nephrol Dial Transpl. 2015;30:1825-33.

36. Nasr SH, Galgano SJ, Markowitz GS, Stokes MB, D'Agati VD. Immunofluorescence on pronase-digested paraffin sections: a valuable salvage technique for renal biopsies. Kidney Int. 2006;70:2148-51.

37. Iatropoulos P, Daina E, Curreri M, Piras R, Valoti E, Mele C, et al. Cluster analysis identifies distinct pathogenetic patterns in C3 glomerulopathies/immune complex-mediated membranoproliferative GN. J Am Soc Nephrol. 2018;29:283-94. 